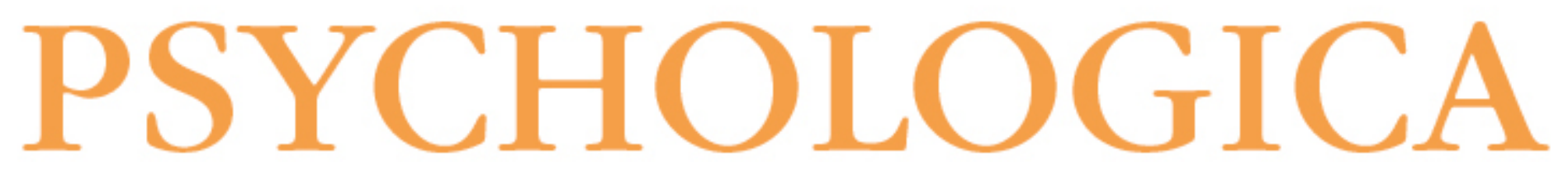

\title{
Resiliência, stresse parental e sobrecarga de pais de crianças com diagnóstico de anomalia congénita
}

\author{
Autor(es): $\quad$ Albuquerque, Sara; Pereira, Marco; Fonseca, Ana; Canavarro, Maria \\ Cristina
}

Publicado por: Imprensa da Universidade de Coimbra

URL

persistente: URI:http://hdl.handle.net/10316.2/39210

DOI: DOI:http://dx.doi.org/10.14195/1647-8606_582_1

Accessed : $\quad$ 26-Apr-2023 15:11:33

A navegação consulta e descarregamento dos títulos inseridos nas Bibliotecas Digitais UC Digitalis, UC Pombalina e UC Impactum, pressupõem a aceitação plena e sem reservas dos Termos e Condições de Uso destas Bibliotecas Digitais, disponíveis em https://digitalis.uc.pt/pt-pt/termos.

Conforme exposto nos referidos Termos e Condições de Uso, o descarregamento de títulos de acesso restrito requer uma licença válida de autorização devendo o utilizador aceder ao(s) documento(s) a partir de um endereço de IP da instituição detentora da supramencionada licença.

Ao utilizador é apenas permitido o descarregamento para uso pessoal, pelo que o emprego do(s) título(s) descarregado(s) para outro fim, designadamente comercial, carece de autorização do respetivo autor ou editor da obra.

Na medida em que todas as obras da UC Digitalis se encontram protegidas pelo Código do Direito de Autor e Direitos Conexos e demais legislação aplicável, toda a cópia, parcial ou total, deste documento, nos casos em que é legalmente admitida, deverá conter ou fazer-se acompanhar por este aviso.

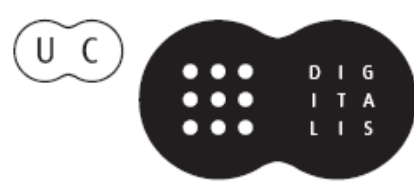


VOLUME $5 \bigcirc$ No2 2015

\section{PSYCHOLOGICA}

IMPRENSA DA UNIVERSIDADE DE COIMBRA

COIMBRA UNIVERSITY PRESS

FACULDADE DE PSICOLOGIA E DE CIÊNCIAS

DA EDUCAÇÃO DA UNIVERSIDADE DE COIMBRA 


\title{
Resiliência, stresse parental e sobrecarga de pais de crianças com diagnóstico de anomalia congénita ${ }^{1}$
}

\author{
Sara Albuquerque ${ }^{2}$, Marco Pereira ${ }^{3}$, Ana Fonseca ${ }^{4}$ e \\ Maria Cristina Canavarro 5
}

Resilience, parenting stress and burden of parents of children with a diagnosis of a congenital anomaly

\begin{abstract}
The purpose of this study was to assess the parental adaptation, in terms of perceived burden and parenting stress, and to examine the potential influence of parents' resilience characteristics in their adaptation. The sample consisted of 45 couples, whose child had a diagnosis of disability or congenital anomaly. The assessment protocol included the Resilience Scale for Adults (RSA), the Impact on Family Scale (IOF) and the Parenting Stress Index - Short form (PSI-SF). The results showed that mothers reported significantly higher scores on Parental distress and fathers reported higher scores on Dysfunctional interaction parents-child. Regarding perceived burden, no significant differences were found between mothers and fathers. Among both parents, resilience was associated with better adaptation outcomes. Maternal resilience was not significantly associated with the paternal adaptation; however, it was observed that higher levels of paternal resilience

1 Este estudo foi desenvolvido no âmbito da linha de investigação Relações, Desenvolvimento e Saúde, da Unidade I\&D Instituto de Psicologia Cognitiva, Desenvolvimento Vocacional e Social (PEst-OE/PSI/UI0192/2011). Marco Pereira é apoiado por uma Bolsa de Pós-Doutoramento da Fundação para a Ciência e Tecnologia (FCT) (SFRH/BPD/44435/2008) e Sara Albuquerque e Ana Fonseca são apoiadas por uma Bolsa de Doutoramento da FCT (SFRH/BD/86223/2012 e SFRH/BD/47053/2008, respetivamente).

2 Faculdade de Psicologia e de Ciências da Educação da Universidade de Coimbra. Email: saramagalhaes9@msn.com

3 Faculdade de Psicologia e de Ciências da Educação da Universidade de Coimbra. Email: marcopereira@fpce.uc.pt

4 Faculdade de Psicologia e de Ciências da Educação da Universidade de Coimbra. Email: anadfonseca@fpce.uc.pt

5 Faculdade de Psicologia e de Ciências da Educação da Universidade de Coimbra. Email: mccanavarro@fpce.uc.pt
\end{abstract}


were significantly associated with better maternal adaptation. Findings from this study highlight that resilience characteristics may be important determinants in the parental adaptation. These findings also emphasize the need to assess not only mothers and fathers separately, but also the couple as a unit of analysis, as well as analyse parental resources and the potential cross-over effects when designing therapeutic interventions.

Keywords: disability; resilience; burden; parenting stress

\section{Resumo}

O presente estudo teve como objetivo avaliar a adaptação parental (em termos de perceção de sobrecarga e stresse parental), bem como o papel das características de resiliência de pais e mães na adaptação parental. A amostra foi constituída por 45 casais, pais de crianças com um diagnóstico de deficiência/anomalia congénita. O protocolo de avaliação incluiu a Escala de Resiliência para Adultos (ERA), a Escala de Impacto Familiar (EIF) e o Índice de Stress Parental (Forma reduzida) (ISP). Os resultados mostraram que as mães apresentaram valores mais elevados de Distress parental e os pais valores mais elevados na dimensão Interação disfuncional pais-criança. Relativamente à sobrecarga, não se verificaram diferenças significativas entre mães e pais. Em ambos os progenitores, a resiliência mostrou-se associada a melhor adaptação. A resiliência materna não se mostrou associada à adaptação paterna, mas constatou-se que níveis elevados de resiliência paterna se associaram significativamente a melhor adaptação materna. As características resilientes revelaram-se como importantes determinantes na adaptação parental. Estes resultados enfatizam ainda a necessidade não só de avaliar os pais e as mães separadamente, mas também o casal como unidade de análise, e de analisar os recursos parentais e os potenciais efeitos cruzados no desenho de intervenções terapêuticas.

Palavras-chave: deficiência; resiliência; sobrecarga; stresse parental

\section{INTRODUÇÃO}

No domínio da Psicologia, as investigações sobre a resiliência têm-se centrado no estudo do desenvolvimento humano, particularmente em populações consideradas de risco (Cicchetti \& Cohen, 1995). Especificamente, procura-se compreender o que está subjacente ao potencial saudável, ou seja, à adaptação positiva que alguns 
indivíduos apresentam em situações de adversidade. Apesar do interesse crescente em estudar a resiliência no âmbito familiar, este tópico tem sido pouco explorado em famílias de crianças com diagnóstico de deficiência ou de anomalia congénita (AC) (Bayat, 2007), tornando-se fundamental investigar os fatores que contribuem para os pais se adaptarem de forma mais positiva a um contexto de adversidade. De igual modo, e apesar de as mães permanecerem como as principais cuidadoras na família, só recentemente os pais começaram a ganhar maior proeminência na investigação na área da família (Day, Lewis, O’Brien, \& Lamb, 2005). Neste sentido, o presente estudo tem como principais objetivos avaliar a importância das características de resiliência de pais e mães de crianças com um diagnóstico de deficiência/AC no stresse parental e na perceção de sobrecarga.

Por definição, a resiliência refere-se à capacidade de adaptação, funcionamento positivo ou competência dos indivíduos, quando na presença de adversidade (Luthar, 2006; Masten, 2007; Rutter, 1990). Nesta linha, o nascimento de uma criança com deficiência/AC é um acontecimento imprevisto que acarreta exigências acrescidas de prestação de cuidados, podendo constituir para os pais um fator de adversidade e indutor de stresse. Porém, investigações mais recentes indicam que muitas das famílias de crianças com deficiência/AC conseguem adaptar-se de forma positiva a esta realidade (Bayat, 2007; Hastings, Allen, McDermott, \& Still, 2002). Para Patterson (2002), muitas famílias superam crises através de um processo de adaptação, resultando em funcionamento competente, que não seria muito diferente do funcionamento das famílias de crianças sem deficiência (Seltzer, Greenberg, Floyd, Pettee, \& Hong, 2001).

Do ponto de vista conceptual, o conceito de resiliência pode ser tomado por dois pontos de vista distintos: a resiliência como resultado, ou seja, o funcionamento adaptativo após a vivência de uma situação de risco e a resiliência como recurso, como fator de proteção em situações indutoras de stresse, potenciando a adaptação em situações traumáticas e adversas (Hjemdal, Friborg, Stiles, Rosenvinge, \& Martinussen, 2006; Masten, 2007). Seguindo esta última conceptualização, a resiliência pode ser entendida como um conjunto de traços de personalidade, qualidades e competências individuais relativamente estáveis que estão associadas à capacidade de superar e ultrapassar a adversidade e lidar com o stresse (Connor \& Davidson, 2003), constituindo assim os processos que operam na produção de saúde na presença de risco (Bianchini \& Dell’Aglio, 2006). É nesta abordagem que centramos o presente estudo.

Perante a ocorrência de um diagnóstico de deficiência/AC num filho, a família vê-se confrontada com a necessidade de reorganização e de redefinição dos papéis familiares, para além de que os pais necessitam de lidar com as diferentes exigências médicas, emocionais, sociais e financeiras associadas ao diagnóstico (Baker-Ericzen, Brookman-Frazee, \& Stahmer, 2005; Mazer et al., 2008; Seligman 
\& Darling, 2007). Todas estas exigências podem implicar maior stresse parental e aduzir significativos níveis de sobrecarga.

O stresse parental tem sido conceptualizado de múltiplas perspetivas, no entanto, com frequência, engloba os desafios e exigências diários associados à prestação de cuidados (Crnic \& Low, 2002). O estudo do stresse parental no contexto de doença/deficiência de um filho é fundamental (Dyson, 1997), dado que se sugere que este desempenha um papel crítico no desenvolvimento das competências parentais e, subsequentemente, no desenvolvimento e bem-estar psicológico da criança (Deater-Deckard, 2005). Neste contexto, os estudos têm mostrado que os pais de crianças com um diagnóstico de deficiência/AC, comparativamente aos pais de crianças saudáveis, têm um risco aumentado de apresentar níveis elevados de stresse parental (Baker-Ericzen et al., 2005; Dyson, 1997; Pelchat et al., 1999; Pope, Tillman, \& Snyder, 2005; Uzark \& Jones, 2003).

O impacto familiar deste tipo de diagnóstico traduz-se também nas exigências acrescidas na prestação de cuidados (Crnic, Friedrich, \& Greenberg, 1983; McKinney \& Peterson, 1987) e em dificuldades financeiras adicionais (Parish, Seltzer, Greenberg, \& Floyd, 2004). Neste contexto, um dos conceitos que tem sido mais estudado é o conceito de sobrecarga (do inglês burden), isto é, os efeitos negativos que decorrem da prestação de cuidados a uma criança com um diagnóstico de deficiência (Brannan, Heflinger, \& Bickman, 1997). Também neste domínio, quando comparados com pais de crianças saudáveis, os estudos têm mostrado que estes pais tendem a vivenciar níveis de sobrecarga mais elevados (Green, 2007; Maes, Broekman, Dosen, \& Nauts, 2003).

Em termos gerais, existe evidência suficiente para esperar que o stresse de cuidar de uma criança com deficiência/AC afete de forma diferente mães e pais (Crnic \& Booth 1991; Creasey \& Reese 1996; McDonald \& Almeida 2004), ainda que alguns autores (e.g., Gray, 2003; Hastings, 2003) refiram que os efeitos do género parental são mais complexos. Comparativamente aos pais, tem sido indicado que as mães apresentam valores mais elevados de stresse (Dellve, Samuelsson, Tallborn, Fasth, \& Hallberg, 2006; Herring et al., 2006), ainda que outros estudos tenham sugerido mais semelhanças do que diferenças entre pais e mães (e.g., Dyson, 1997; Keller \& Honig, 2004; Saloviita, Itälinna, \& Leinonen, 2003). Relativamente à prestação de cuidados, os estudos têm sustentado que as mães, comparativamente aos pais, experienciam níveis superiores de sobrecarga (e.g., Beckman, 1991; Heller, Hsieh, \& Rowitz, 1997), principalmente pelo facto de estas assumirem a maior parte da prestação de cuidados à criança (Moes, Koegel, Schreibman, \& Loos, 1992). Porém, alguns estudos (e.g., Hunfeld, Tempels, Passchier, Hazebroek, \& Tibboel, 1999; Albuquerque, Pereira, Fonseca, \& Canavarro, 2012), não encontraram diferenças entre pais e mães nesta variável. Mais recentemente, alguns autores têm salientado a existência de interde- 
pendência diádica, destacando como os atributos e comportamentos de um membro da díade poderão influenciar a adaptação do outro (Campbell \& Kashy, 2002). No contexto da deficiência, destaca-se um estudo no qual foi salientada a interdependência em termos de influência dos indicadores de adaptação individual de um membro do casal no outro (Gerstein, Crnic, Blacher, \& Baker, 2009).

No que se prende com a associação entre a resiliência e os indicadores de adaptação parental, a generalidade dos estudos tem-se centrado em conceitos próximos ao de resiliência (e.g., o conceito de robustez) ou no papel que os recursos pessoais e familiares desempenham na adaptação aos desafios subjacentes ao cuidar de uma criança com deficiência (e.g., Blacher \& Baker, 2007; Heiman, 2002; Perry, 2005). Por exemplo, Failla e Jones (1991) verificaram que a robustez familiar atuava como uma fonte de resistência, conduzindo a uma diminuição dos efeitos negativos do stresse, aumentando o acesso ao apoio social e, deste modo, facilitando a adaptação. Entre os recursos familiares, a investigação tem-se centrado fundamentalmente em variáveis como o apoio social, a estrutura e coesão familiar (e.g., Dyson, 1997; Fonseca, Nazaré, \& Canavarro, 2014; Saloviita et al., 2003; Taanila, Jarvelin, \& Kokkonen, 1999; Tak \& McCubbin, 2002), sugerindo-se que estas variáveis operam como fatores de resiliência no ajustamento parental. A este respeito, Dyson (1997) reportou uma associação negativa entre os recursos psicológicos familiares (e.g., enfâse da família no crescimento pessoal, coesão familiar e apoio familiar) e o stresse parental.

Face ao exposto, é importante que a investigação nesta área se estenda também ao domínio da parentalidade, dado que as características resilientes dos pais em situações de stresse podem ter um impacto positivo no desenvolvimento da criança, modelando formas positivas de lidar com adversidades. Neste sentido, o presente estudo tem como objetivos: (1) investigar as diferenças de género na adaptação parental (stresse parental e sobrecarga); e (2) examinar o papel das características resilientes dos pais na adaptação parental do próprio e do(a) parceiro(a).

\section{MÉTODO}

\section{Participantes}

A amostra deste estudo foi composta por 90 participantes (45 casais), pais de crianças com um diagnóstico de anomalia congénita. Foram considerados critérios de inclusão para o presente estudo: a) casais que foram pais de uma criança com 
um diagnóstico pré-natal ou pós-natal de AC e que estavam casados ou a viver em união de facto; b) idade dos pais superior a 18 anos; e c) um nível de compreensão de Português adequado para o preenchimento dos questionários que compõem a bateria de avaliação.

No Quadro 1 encontram-se as características sociodemográficas da amostra. Globalmente, pais e mães tinham uma idade média de 37 anos e a duração média da relação conjugal era de cerca de 11 anos. A maioria dos pais vivia em meio urbano (53.3\%) e encontravam-se ambos empregados (pais: $95.5 \%$; mães: $88.6 \%$ ). Os pais e mães da presente amostra não se distinguiram em nenhuma das variáveis sociodemográficas em análise.

Quadro 1

Caracterização Sociodemográfica da Amostra $(N=45$ Casais $)$

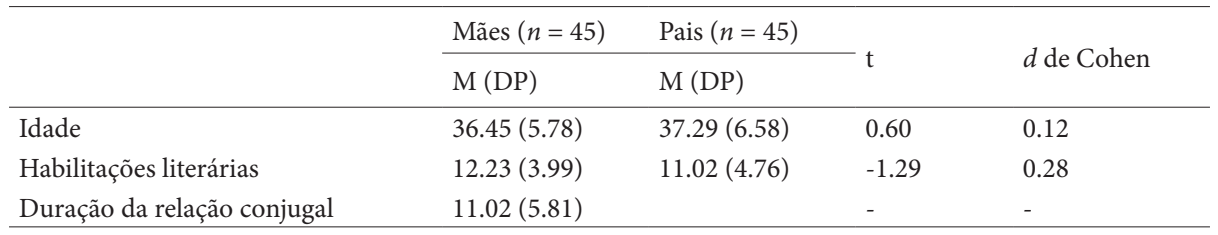

Relativamente às crianças com diagnóstico de $\mathrm{AC}$, estas tinham uma média de idade de 47.64 meses $(D P=35.46)$ e eram maioritariamente do sexo masculino (55.6\%). O diagnóstico foi conhecido no período pré-natal em $62.2 \%$ dos casos e os diagnósticos mais frequentes foram as anomalias cromossómicas (35.6\%), seguido das uropatias (28.6\%), das cardiopatias (11.9\%) e das anomalias dos sistemas digestivo $(9.5 \%)$ e respiratório $(4.8 \%)$.

\section{Instrumentos}

A bateria de avaliação utilizada no presente estudo foi composta por duas fichas de dados e pelos instrumentos de autorresposta descritos em seguida:

Ficha de dados sociodemográficos e ficha de dados relativos ao diagnóstico. A primeira incluía informação sobre dados sociodemográficos gerais, situação relacional e contexto familiar. A segunda incluía informações sobre as características relativas à criança, incluindo sociodemográficas e clínicas (tipo de AC, momento do diagnóstico, causas da anomalia, ocorrência e número de hospitalizações, tratamentos específicos). O tipo de AC foi classificado tendo em conta o sistema de classificação European Surveillance of Congenital Anomalies (EUROCAT, 2009).

Escala de Resiliência para o Adulto (ERA; Friborg, Hjemdal, Rosenvinge, \& Martinussen, 2003; Pereira, Cardoso, Alves, Narciso, \& Canavarro, 2013 
- Versão Portuguesa; VP). A ERA contém itens relacionados com cinco componentes da resiliência: Competências pessoais, Coesão familiar, Recursos sociais, Competências sociais e Estilo estruturado. A ERA é composta por 33 itens com respostas de diferenciação semântica, de modo a reduzir a tendência para a aquiescência e permite avaliar os recursos protetores que promovem a resiliência na idade adulta. Cada item é cotado numa escala de resposta de 1 a 7 , com resultados mais elevados a refletir maior nível de resiliência. Na presente amostra os valores de a de Cronbach variaram entre .42 (Estilo estruturado - mães) e .84 (Coesão familiar - mães). Devido à baixa consistência interna do fator Estilo estruturado, este não foi utilizado nas análises estatísticas.

Parenting Stress Index - Índice de Stress Parental - Forma reduzida (ISP; Abidin, 1993; Santos, 2011 - VP). O ISP é composto por 36 itens, respondidos numa escala de resposta de 5 pontos, desde 1 (Concordo completamente) a 5 (Discordo completamente). O ISP procura avaliar o stresse percecionado pelos pais no exercício do seu papel parental (e não por acontecimentos de vida indutores de stresse independentes desse papel) e organiza-se em três domínios: Distress parental (nível de stresse resultante das exigências de educar uma criança), Interação disfuncional pais-criança (avalia a insatisfação parental com as interações com a criança) e Criança difícil (avalia as perceções parentais das capacidades autorreguladoras da criança). Na presente amostra os valores de consistência interna variaram entre .82 (Interação disfuncional mãe/pai-criança - mães) e .87 (Distress parental - pais).

Escala de Impacto Familiar (EIF; Stein \& Jessop, 2003; Albuquerque, Fonseca, Pereira, Nazaré, \& Canavarro, 2011 - VP). A EIF consiste numa medida do impacto da doença/deficiência da criança na família, que avalia a sobrecarga (impacto familiar global) de uma forma unidimensional. É constituída por 15 itens, respondidos numa escala de resposta de quatro pontos, desde 1 (Discordo muito) a 4 (Concordo muito). No presente estudo, e de acordo com as recomendações dos autores, um conjunto adicional de quatro itens que avaliam a sobrecarga financeira foi também utilizado. Na presente amostra, o valor do a de Cronbach foi de .89 para as mães e .91 para os pais. A consistência interna do fator de sobrecarga financeira foi de .80 para as mães e .84 para os pais.

\section{Procedimentos}

A amostra do presente estudo foi recolhida de dois modos distintos. Em primeiro lugar, foi realizado um levantamento das utentes da Maternidade Doutor 
Daniel de Matos do Centro Hospitalar e Universitário de Coimbra (MDM-CHUC) que receberam o diagnóstico de AC no bebé no período pré ou pós natal, sendo que o diagnóstico de deficiência/anomalia congénita tinha ocorrido há pelo menos seis meses. Aos casais que preenchiam os critérios de inclusão, foi enviada uma carta, solicitando o preenchimento do protocolo de investigação e o reenvio dos protocolos, já preenchidos, para a MDM-CHUC. Por outro lado, foram efetuadas pesquisas, nomeadamente em fóruns de debate e partilha sobre a temática, e contou-se ainda com a colaboração das seguintes associações de apoio a pais de crianças com Trissomia 21: a Associação Olhar 21, a Associação Portuguesa de Portadores de Trissomia 21 (APPT 21) e a Associação de Viseu de Portadores de Trissomia 21 (AVISPT 21). Aos pais que preenchiam os critérios de inclusão, e que mostraram disponibilidade para participar na investigação, foi pedido que enviassem a sua morada, tendo o restante processo sido realizado de modo semelhante às utentes da MDM-CHUC. No total, foram enviados 244 protocolos de avaliação (122 casais), tendo 144 retornado preenchidos, o que correspondeu a uma taxa de resposta de 59\%. Foram excluídos 10 protocolos porque apenas a mãe preencheu os questionários e excluíram-se 44 protocolos referentes a 22 casais que não preencheram completamente os instrumentos da bateria de avaliação.

Todos os participantes foram informados sobre os objetivos do estudo, tendo sido pedido que respondessem de forma espontânea e sincera e tendo sido assegurada a confidencialidade e anonimato das respostas aos questionários. O presente estudo foi aprovado pela Comissão de Ética do Centro Hospitalar e Universitário de Coimbra, EPE.

\section{Análises estatísticas}

O tratamento estatístico dos dados foi realizado através do programa Statistical Package for the Social Sciences (SPSS - v. 17.0). Recorreu-se ao teste do Qui-Quadrado para comparação de dados categoriais e ao teste $t$ de Student para comparação dos grupos nas características demográficas de natureza contínua. Para comparação de médias entre os grupos recorremos à análise multivariada da variância (MANOVA) de Medidas Repetidas, considerando a variável género como fator intrasujeitos (considerando a interdependência entre os membros do casal) e os indicadores de stresse parental e sobrecarga como variáveis dependentes. Adicionalmente, foram calculados coeficientes de correlação de Pearson para avaliar a associação entre variáveis de natureza contínua. A magnitude dos efeitos foi analisada através do $d$ de Cohen e o $V$ 
de Cramer, adotando as convenções seguintes: efeito pequeno: $0.20 \geq d<0.50$, $.10 \geq V<.30$; efeito médio: $0.50 \geq d<0.80, .30 \geq V<.50$; efeito grande: $d \geq$ $0.80, V \geq .50)$ (Cohen, 1992).

\section{RESULTADOS}

\section{Caracterização do stresse parental e da perceção de sobrecarga parental}

No Quadro 2 são apresentadas as estatísticas descritivas dos indicadores de stresse parental e perceção de sobrecarga. Em relação ao stresse parental verificou-se um efeito multivariado estatisticamente significativo da variável género [Lambda de Wilks $=0.64, F_{(3,42)}=7.81, p<.001, \eta_{p}^{2}=.36$ ]. Os testes univariados subsequentes mostraram efeitos significativos nos fatores Distress parental e Interação disfuncional pais-criança. Pela análise dos valores médios podemos verificar que as mães apresentam valores mais elevados na dimensão Distress parental, enquanto na dimensão Interação disfuncional pais-criança são os pais que apresentam valores mais elevados. Quanto à sobrecarga parental, o efeito multivariado não se mostrou estatisticamente significativo [Lambda de Wilks $\left.=0.97, F_{(2,43)}=0.58, p=.564, \eta_{\mathrm{p}}^{2}=.03\right]$.

Quadro 2

Comparação do Stresse Parental e da Perceção de Sobrecarga Parental

\begin{tabular}{lllll}
\hline & Mães $(n=45)$ & Pais $(n=45)$ & & \\
\cline { 2 - 3 } & M (DP) & M (DP) & & \\
& & & & \\
\hline Stresse parental & $27.88(8.44)$ & $24.51(7.63)$ & $6.28^{*}$ & .13 \\
$\quad$ Distress parental & ${ }^{2}$ \\
$\quad$ Interação disfuncional mãe/pai-criança & $18.84(5.90)$ & $21.04(6.65)$ & $5.00^{*}$ & .10 \\
Criança difícil & $22.55(7.28)$ & $24.60(7.40)$ & 3.45 & .07 \\
$\quad$ Stresse total & $69.27(16.22)$ & $70.15(18.40)$ & 0.11 & .00 \\
Impacto familiar & & & & \\
$\quad$ Sobrecarga global & $26.13(7.70)$ & $25.61(7.43)$ & 0.31 & .01 \\
$\quad$ Sobrecarga financeira & $7.58(3.14)$ & $7.71(2.78)$ & 0.12 & .00 \\
\hline
\end{tabular}

${ }^{*} p<.05$

${ }^{* *} p<.01$

${ }^{* * *} p<.001$ 
Associação entre resiliência, stresse parental e perceção de sobrecarga

Nos Quadros 3 e 4 apresentam-se, separadamente para pais e mães, os valores das correlações entre as características de resiliência e os indicadores de stresse parental e perceção de sobrecarga (global e financeira).

Em relação à associação entre resiliência materna e os indicadores de adaptação materna, globalmente, uma maior resiliência materna mostrou-se correlacionada com menor Distress parental, Stresse total e Sobrecarga global. O fator Coesão familiar correlacionou-se negativamente com a perceção de Sobrecarga financeira. Em relação à associação da resiliência paterna com as dimensões de adaptação materna, foi possível verificar que níveis elevados de resiliência paterna (em particular, Coesão familiar e Competências sociais) mostraram-se correlacionados com menor Distress parental e menores Sobrecargas global e financeira.

Quadro 3

Correlação entre as Características de Resiliência Materna e Paterna e o Stresse e Perceção de Sobrecarga Maternos

\begin{tabular}{|c|c|c|c|c|c|c|}
\hline & \multicolumn{4}{|c|}{ Stresse parental } & \multicolumn{2}{|c|}{ Perceção de sobrecarga } \\
\hline & $\mathrm{DP}$ & IDC & $\mathrm{CD}$ & Total & Global & Financeira \\
\hline \multicolumn{7}{|l|}{ Resiliência (Mãe) } \\
\hline Competência Pessoal & $-.61^{\star \star \star}$ & -.15 & -.11 & $-.42^{\star *}$ & $-.32^{*}$ & -.24 \\
\hline Coesão Familiar & $-.40^{\star \star}$ & -.18 & -.27 & $-.39 * *$ & $-.36^{*}$ & $-.37^{\star}$ \\
\hline Recursos Sociais & $-.40^{\star \star}$ & -.05 & -.15 & -.29 & -.19 & -.22 \\
\hline Competências Sociais & -.22 & -.04 & -.14 & -.19 & -.19 & -.07 \\
\hline \multicolumn{7}{|l|}{ Resiliência (Pai) } \\
\hline Competência Pessoal & -.21 & -.14 & -.09 & -.20 & $-.36^{*}$ & -.16 \\
\hline Coesão Familiar & $-.32^{*}$ & -.14 & -.24 & $-.33^{\star}$ & $-.48^{\star \star}$ & $-.38^{\star *}$ \\
\hline Recursos Sociais & -.24 & -.02 & -.10 & -.16 & -.23 & -.14 \\
\hline Competências Sociais & $-.46^{\star *}$ & -.13 & -.15 & $-.35^{\star}$ & $-.51^{\star * \star}$ & $-.36^{*}$ \\
\hline
\end{tabular}

DP: Distress Parental; IDC: Interação Disfuncional Pais-Criança; CD: Criança Difícil ${ }^{*} p<.05$

${ }^{* *} p<.01$

${ }^{* * *} p<.001$

No que respeita à associação entre a resiliência paterna e os indicadores de stresse parental e perceção de sobrecarga, constatou-se um padrão semelhante ao observado para a mãe, com a maioria das associações a observarem-se com a maioria dos indicadores de stresse parental (com exceção do fator Criança difícil) e perceção de Sobrecargas global e financeira. A resiliência materna não se mostrou significativamente correlacionada com os indicadores paternos. 
Quadro 4

Correlação entre as Características de Resiliência Materna e Paterna e o Stresse e Perceção de Sobrecarga Paternos

\begin{tabular}{|c|c|c|c|c|c|c|}
\hline & \multicolumn{4}{|c|}{ Stresse parental } & \multicolumn{2}{|c|}{ Perceção de sobrecarga } \\
\hline & DP & IDC & $\mathrm{CD}$ & Total & Global & Financeira \\
\hline \multicolumn{7}{|l|}{ Resiliência (Mãe) } \\
\hline Competência Pessoal & -.29 & -.05 & -.05 & -.16 & -.14 & -.07 \\
\hline Coesão Familiar & $-.30^{*}$ & -.09 & -.09 & -.19 & -.24 & -.28 \\
\hline Recursos Sociais & $-.34^{\star}$ & .04 & .04 & -.11 & -.13 & -.20 \\
\hline Competências Sociais & .00 & .19 & -.02 & -.06 & .21 & .14 \\
\hline \multicolumn{7}{|l|}{ Resiliência (Pai) } \\
\hline Competência Pessoal & $-.68^{\star * *}$ & $-.39^{\star *}$ & -.11 & $-.47^{\star \star}$ & $-.46^{\star *}$ & $-.31^{\star}$ \\
\hline Coesão Familiar & $-.58^{\star \star \star}$ & $-.45^{\star *}$ & -.28 & $-.52^{\star \star \star}$ & $-.58^{\star * *}$ & $-.40^{\star *}$ \\
\hline Recursos Sociais & $-.57^{\star \star \star}$ & -.22 & -.18 & $-.39^{\star \star}$ & $-.36^{*}$ & $-.30^{*}$ \\
\hline Competências Sociais & $-.70^{\star * *}$ & $-.43^{\star *}$ & -.27 & $-.55^{\star \star *}$ & $-.62^{\star * *}$ & $-.50^{\star *}$ \\
\hline
\end{tabular}

DP: Distress parental; IDC: Interação disfuncional com a criança; CD: Criança difícil

${ }^{*} p<.05$;

${ }^{* *} p<.01 ;$

${ }^{* * *} p<.001$

\section{DISCUSSÃO}

No presente estudo procurámos assumir uma perspetiva não-determinista do impacto da presença de uma criança com um diagnóstico de deficiência/AC na adaptação familiar. De forma congruente com esta perspetiva, os nossos resultados denotam que as características resilientes dos pais estão associadas a uma adaptação parental mais positiva, sugerindo que apesar do confronto com uma experiência adversa como o diagnóstico de deficiência/AC na criança, as famílias conseguem resistir e recuperar da crise e sofrimento associado, adaptando-se a esta realidade.

No que respeita ao stresse parental, foram encontradas algumas diferenças entre pais e mães, ainda que não se tenham registado diferenças no valor total. Primeiro, verificou-se que as mães apresentam valores mais elevados de Distress parental, aspeto congruente com os resultados dos estudos revistos (Dellve et al., 2006; Herring et al., 2006) e que poderá ser explicado pelo facto de estas assumirem o principal papel na prestação de cuidados à criança. Pelo contrário, na dimensão Interação disfuncional pais-criança, são os pais que apresentam valores mais elevados. No âmbito da parentalidade, alguns autores salientam que pais resilientes têm melhores relacionamentos com seus filhos e são mais consistentes no controlo do seu comportamento (Bynum \& Brody, 2005). Neste sentido, e tendo 
também em consideração as correlações negativas e estatisticamente significativas encontradas entre as dimensões de resiliência paternas e este indicador de stresse parental, é possível que os pais menos resilientes possam ter maiores dificuldades e perceber como mais difícil a interação com os filhos. Em acréscimo, Shonkoff, Hauser-Cram, Krauss e Upshur (1992) salientaram as dificuldades ao nível da vinculação como uma das principais fontes de stresse, em particular para os pais, destacando também como consequência desta variável as dificuldades na interação com a criança, o que poderá explicar os resultados encontrados.

Relativamente à perceção de sobrecarga, não se verificaram diferenças significativas entre as mães e os pais, resultado que, apesar de contrário à maioria da literatura revista (e.g., Beckman, 1991; Heller et al., 1997), é consistente com estudos que sugerem que, ainda que o impacto associado ao diagnóstico se possa manifestar diferencialmente em termos de stresse parental, afere-se uma tendência para os membros do casal a avaliar esse impacto e sobrecarga de forma semelhante (Albuquerque et al., 2012; Hunfeld et al., 1999). Estes resultados poderão ser explicados pela evolução dos papéis parentais devido a um rápido aumento do número de esposas e mães no mercado de trabalho (Yeung, Sandberg, Davis-Kean, $\&$ Hofferth, 2001), aspeto que se manifesta na presente amostra, encontrando-se a maior parte dos pais ambos empregados, o que poderá obrigar a uma divisão mais equilibrada das tarefas parentais.

Os nossos resultados evidenciam ainda que, tanto para os pais como para as mães, quanto maiores os níveis de resiliência, melhor a sua adaptação, isto é, menor o stresse parental, bem como menor a perceção de impacto familiar/sobrecarga associada ao diagnóstico do filho. Estes resultados fundamentam a adoção do conceito de resiliência enquanto recurso, como fator de proteção em situações indutoras de stresse, potenciando a adaptação em situações de adversidade, aspeto igualmente replicado noutros estudos (Failla \& Jones, 1991). Em relação à associação entre as características de resiliência de um progenitor e a adaptação do outro, verificámos que, globalmente, a resiliência materna não se mostrou associada à adaptação paterna, mas constatou-se que níveis elevados de resiliência paterna se associam de forma significativa à adaptação materna. Alguns autores têm salientado esta interdependência diádica, também na área da deficiência, embora sobretudo em termos de influência dos indicadores de adaptação individual de um membro do casal no outro (Gerstein et al., 2009). Uma possível explicação para estes resultados é que as características resilientes de um dos membros do casal influenciam a sua adaptação individual que, por sua vez, pode influenciar a adaptação individual do(a) companheiro(a). Estes resultados parecem reforçar o papel da relação conjugal na adaptação e a importância de os homens não serem negligenciados na investigação e na intervenção clínica. Estas hipóteses justificam a necessidade de este tópico ser objeto de investigações futuras. 
Na realização deste estudo destacamos importantes limitações à generalização dos resultados aqui apresentados, as quais se prendem, em primeiro lugar, com a amostra, nomeadamente o seu tamanho relativamente reduzido e o método de amostragem por conveniência. Por outro lado, o recrutamento dos participantes por via de serviços de saúde e associações sociais implica que estes beneficiam de acompanhamento específico, o que poderá traduzir-se numa maior dificuldade em transpor estes resultados para pais de crianças com AC que não tiveram acesso a estes serviços e recursos. Igualmente, a taxa de participação, embora consideremos ser devida ao procedimento utilizado para solicitar a participação no estudo (envio dos questionários por correio, ausência de contato presencial), deve ser um fator a ter em conta no que respeita a generalização dos resultados. A nível metodológico, a natureza transversal da presente investigação constitui também uma limitação, uma vez que impossibilita uma avaliação da adaptação parental ao longo do tempo, salientando-se desta forma a pertinência de se delinearem projetos de investigação longitudinal.

Apesar destas limitações, estes resultados permitiram-nos constatar que, não descurando o impacto nestes pais do diagnóstico de deficiência/AC da criança, com todos as exigências e desafios que este acarreta, encontramos em muitos a presença de características resilientes, o que questiona, em certa medida, a orientação excessivamente centrada numa vertente "psicopatológica", que assume uma reação linear entre a notícia do diagnóstico de deficiência/AC de um filho e a perturbação do funcionamento parental. Não obstante, é importante ressalvar que um modelo mais positivo em relação à forma como a deficiência da criança é percecionada, não significa que devemos ignorar a existência de tensões e desafios envolvidos na prestação de cuidados. Adicionalmente, este estudo poderá dar um importante contributo para o conhecimento da adaptação parental, materna e paterna, ao nascimento de um filho com deficiência/AC, em particular, ao nível da resiliência e capacidade pessoal de adaptação parental, aspetos que têm sido pouco explorados na investigação nacional. Nesta linha, salientamos a inclusão do pai, elemento ainda muito negligenciado na maioria das investigações nesta área. Consideramos ainda relevante destacar a utilização, no presente estudo, do casal enquanto unidade de análise, aspeto que tem igualmente vindo a ser salientado por alguns autores. Com efeito, Svavarsdottir e Rayens (2005) salientam que ao abarcar os processos e a interdependência na díade, poderemos obter uma compreensão mais profícua do funcionamento e mecanismos de adaptação dos casais e famílias quando confrontados com adversidades, como por exemplo, o cuidar de uma criança com AC.

Finalmente, os resultados deste estudo permitem-nos ainda tecer alguns comentários acerca da intervenção, na área clínica, junto dos casais que tenham 
um filho com deficiência/AC. Com efeito, como referido por Trute e Hauch (1988), se se estudarem as características e atributos dos pais que, apesar de um contexto adverso, apresentam bons indicadores de adaptação, então, será possível compreender e intervir junto dos pais em maior risco de inadaptação. Assim, o foco nos recursos parentais, permite-nos apreender a diversidade de reações parentais perante o diagnóstico, permitindo, deste modo, a identificação de situações de risco psicológico, que necessitem de uma intervenção mais específica e individualizada. O delineamento de estratégias que reforcem as competências pessoais e sociais, mas também os recursos sociais e a coesão familiar, com vista à promoção da resiliência parental pode ser o ponto inicial da intervenção por parte dos profissionais de saúde no apoio a estas famílias. Por outro lado, as associações encontradas entre as características de resiliência de um progenitor e a adaptação do outro, particularmente das características de resiliência paternas na adaptação das mães, poderão despertar os profissionais para a importância do papel da relação conjugal na adaptação e do contexto interpessoal em que esta problemática ocorre.

\section{REFERÊNCIAS}

Abidin, R. (1983). Parenting stress index (PSI). Charlottesville, VA: Pediatric Psychology Press.

Albuquerque, S., Fonseca, A., Pereira, M., Nazaré, B., \& Canavarro, M. C. (2011). Estudos psicométricos da versão portuguesa da Escala de Impacto Familiar (EIF). Laboratório de Psicologia, 9(2), 173-187.

Albuquerque, S., Pereira, M., Fonseca, A., \& Canavarro, M. C. (2012). Impacto familiar e ajustamento de pais de crianças com diagnóstico de anomalia congénita: Influência dos determinantes da criança. Revista de Psiquiatria Clínica, 39(4), 136-141.

Baker-Ericzen, M. J., Brookman-Frazee, L., \& Stahmer, L. (2005). Stress levels and adaptability in parents of toddlers with and without autism spectrum disorders. Research \& Practice for Persons with Severe Disabilities, 30(4), 194-204.

Bayat, M. (2007). Evidence of resilience in families of children with autism. Journal of Intellectual Disability Research, 51(9), 702-704.

Beckman, P. J. (1991). Comparison of mothers' and fathers' perceptions of the effect of young children with and without disabilities. American Journal on Mental Retardation, 95(5), 585-595.

Bianchini, D., C., \& Dell'Aglio, D. D. (2006). Processos de resiliência no contexto de hospitalização: Um estudo de caso. Paidéia, 16(35), 427-436.

Blacher, J., \& Baker, B. L. (2007). Positive impact of intellectual disability on families. American Journal on Mental Retardation, 112(5), 330-348.

Brannan, A. M., Heflinger, C. A., \& Bickman, L. (1997). The caregiver strain questionnaire: Measuring the impact on the family of living with a child with serious emotional disturbance. Journal of Emotional and Behavioral Disorders, 5(4), 212-222. 
Bynum, M. S., \& Brody, G. H. (2005). Coping behaviors, parenting, and perceptions of children's internalization and externalizing problems in rural African-American mothers. Family Relations, 54(1), 58-71.

Campbell, L., \& Kashy, D. A. (2002). Estimating actor, partner, and interaction effects for dyadic data using PROC MIXED and HLM: A user-friendly guide. Personal Relationships, 9(3), 327-342.

Cicchetti, D., \& Cohen, D. J. (1995). Perspectives on developmental psychopathology. In D. Cicchetti \& D. J. Cohen (Eds.), Developmental psychopathology: Theory and method (Vol. 1, pp. 3-20). New York, NY: Jon Wiley \& Sons, Inc.

Cohen, J. (1992). A power prime. Psychological Bulletin, 112(1), 155-159.

Connor, K. M., \& Davidson, J. R. (2003). Development of a new resilience scale: The Connor-Davidson Resilience Scale (CD-RISC). Depression and Anxiety, 18(2), 76-82.

Creasey, G., \& Reese, M. (1996). Mothers' and fathers' perceptions of parenting hassles: Associations with psychological symptoms, non-parenting hassles, and child behavior problems. Journal of Applied Developmental Psychology, 17(3), 393-406.

Crnic, K. A., \& Booth, C. L. (1991). Mothers' and fathers' perceptions of daily hassles of parenting across early childhood. Journal of Marriage and Family, 53(4), 1042-1050.

Crnic, K. A., Friedrich, W., \& Greenberg, M. (1983). Adaptation of families with mentally retarded children: A model of stress, coping, and family ecology. American Journal of Mental Deficiency, 88(2), 125-138.

Crnic, K. A., \& Low, C. (2002). Everyday stresses and parenting. In M. H. Bornstein (Ed.), Handbook of parenting: Practical issues in parenting (Vol. 5, pp. 243-267). Mahwah, NJ: Erlbaum.

Day, R. D., Lewis, C., O’Brien, M., \& Lamb, M. E. (2005). Fatherhood and father involvement: Emerging constructs and theoretical orientations. In V. Bengtson, A. Acock, K. Allen, P. Dilworth-Anderson, \& D. Klein (Eds.), Sourcebook of family theories (pp. 341-351). London: Sage

Deater-Deckard, K. (2005). Parenting stress and children's development: Introduction to the special issue. Infant and Child Development, 14(2), 111-115.

Dellve, L., Samuelsson, L., Tallborn, A., Fasth, A., \& Hallberg, L. R. (2006). Stress and well-being among parents of children with rare diseases: A prospective intervention study. Journal of Advanced Nursing, 53(4), 392-402.

Dyson, L. I. (1997). Fathers and mothers of school-age children with developmental disabilities: Parental stress, family functioning and social support. American Journal on Mental Retardation, 102(3), 267-279.

EUROCAT (2009). Special report. The status of health in the European Union: Congenital malformations. EUROCAT Central Registry, University of Ulster.

Failla, S., \& Jones, C. (1991). Families of children with developmental disabilities: An examination of family hardiness. Research in Nursing and Health, 14(1), 41-50.

Fonseca, A., Nazaré, B., \& Canavarro, M. C. (2014). The role of satisfaction with social support in perceived burden and stress of parents of six-month-old infants with a congenital anomaly: Actor and partner effects. Journal of Child Health Care, 18(2), 178-191.

Friborg, O., Hjemdal, O., Rosenvinge, J. H., \& Martinussen, M. (2003). A new rating scale for adult resilience: What are the central protective resources behind healthy adjustment? International Journal of Methods in Psychiatric Research, 12(2), 65-76.

Gerstein, E. D., Crnic, K. A., Blacher, J., \& Baker, B. L. (2009). Resilience and the course of daily parenting stress in families of young children with intellectual disabilities. Journal of Intellectual Disability Research, 53(12), 981-997. 
Gray, D. E. (2003). Gender and coping: The parents of children with high functioning autism. Social Science \& Medicine, 56(3), 631-642.

Green, S. E. (2007). "We're tired, not sad": Benefits and burdens of mothering a child with a disability. Social Science \& Medicine, 64(1), 150-163.

Hastings, R. P. (2003). Child behaviour problems and partner mental health as correlates of stress in mothers and fathers of children with autism. Journal of Intellectual Disability Research, 47(4), 231-237.

Hastings, R. P., Allen, R., McDermott, K., \& Still, D. (2002). Factors related to positive perceptions in mothers of children with intellectual disabilities. Journal of Applied Research in Intellectual Disabilities, 15(3), 269-275.

Heiman, T. (2002). Parents of children with disabilities: Resilience, coping and future expectations. Journal of Developmental and Physical Disabilities, 14(2), 159-171.

Heller, T., Hsieh, K., \& Rowitz, L. (1997). Maternal and paternal caregiving of persons with mental retardation across the lifespan. Family Relations, 46(4), 407-415.

Herring, S., Gray, K., Taffe, J., Tonge, B., Sweeney, D., \& Einfeld, S. (2006). Behaviour and emotional problems in toddlers with pervasive developmental disorders and developmental delay: Associations with parental mental health and family functioning. Journal of Intellectual Disability Research, 50(12), 874-882.

Hjemdal, O., Friborg, O., Stiles, T. C., Rosenvinge, J. H., \& Martinussen, M. (2006). Resilience predicting psychiatric symptoms: A prospective study of protective factors and their role in adjustment to stressful life events. Clinical Psychology and Psychotherapy, 13(3), 194-201.

Hunfeld, J. A. M., Tempels, A., Passchier, J., Hazebroek, F. W. J., \& Tibboel, D. (1999). Parental burden and grief one year after the birth of a child with a congenital anomaly. Journal of Pediatric Psychology, 24(6), 515-520.

Keller, D., \& Honig, A. S. (2004). Maternal and paternal stress in families with school-aged children with disabilities. American Journal of Orthopsychiatry, 74(3), 337-348.

Luthar, S. S. (2006). Resilience in development: A synthesis of research across five decades. In D. Cicchetti, \& D. J. Cohen (Eds.), Developmental psychopathology: Risk, disorder, and adaptation ( $2^{\text {nd }}$ ed., Vol. 3, pp. 739-795). Hoboken, NJ: Wiley and Sons.

Maes, B., Broekman, T. G., Dosen, A., \& Nauts, J. (2003). Caregiving burden of families looking after persons with intellectual disability and behavioural or psychiatric problems. Journal of Intellectual Disability Research, 47(6), 447-455.

Masten, A. S. (2007). Resilience in developing systems: Progress and promise as the fourth wave rises. Development and Psychopathology, 19(3), 921-930.

Mazer, P., Gischler, S., Koot, H., Tibboel, D., van Dijk, M., \& Duivenvoorden, H. (2008). Impact of a Child with Congenital Anomalies on Parents (ICCAP) questionnaire: A psychometric analysis. Health and Quality of Life Outcomes, 6, 102-112.

McDonald, D. A., \& Almeida, D. M. (2004). The interweave of fathers' daily work experiences and fathering behaviors. Fathering: A Journal of Theory, Research, \& Practice about Men as Fathers, 2(3), 235-251.

McKinney, B., \& Peterson, R. A. (1987). Predictors of stress in parents of developmentally disabled children. Journal of Pediatric Psychology, 12(1), 133-149.

Moes, D., Koegel, R. L., Schreibman, L., \& Loos, L. M. (1992). Stress profiles for mothers and fathers of children with autism. Psychological Reports, 71, 1272-1274.

Parish, S. L., Seltzer, M. M., Greenberg, J. S., \& Floyd, F. J. (2004). Economic implications of caregiving at midlife: Comparing parents of children with developmental disabilities to other parents. Mental Retardation, 42(6), 413-426. 
Patterson, J. M. (2002). Integrating family resilience and family stress theory. Journal of Marriage and Family, 64(2), 349-360.

Pelchat, D., Ricard, N., Bouchard, J. M., Perrault, M., Saucier, J.-F., Berthiaume, M., \& Bisson, J. (1999). Adaptation of parents in relation to their 6-month-old infant's type of disability. Child: Care, Health \& Development, 25(4), 377-397.

Pereira, M., Cardoso, M., Alves, S., Narciso, I., \& Canavarro, M. C. (2013). Estudos preliminares das características psicométricas da Escala de Resiliência para Adultos (ERA). In A. Pereira, M. Calheiros, P. Vagos, I. Direito, S. Monteiro, C. F. Silva, \& A. Allen Gomes (Eds.), Livro de atas do VIII Simpósio Nacional de Investigação em Psicologia (pp. 93-103). Aveiro: Associação Portuguesa de Psicologia. Consultado em: http://hdl.handle.net/10316/23602

Perry, A. (2005). A model of stress in families of children with developmental disabilities: Clinical and research applications. Journal on Developmental Disabilities, 11(1), 1-16.

Pope, A. W., Tillman, K., \& Snyder, H. (2005). Parenting stress in infancy and psychosocial adjustment in toddlerhood: A longitudinal study of children with craniofacial anomalies. Cleft Palate-Craniofacial Journal, 42(5), 556-559.

Rutter M. (1990). Psychosocial resilience and protective mechanisms. In J. Rolf, A. S. Masten, D. Cicchetti, K. H. Nuechterlein, \& S. Weintraub (Eds.), Risk and protective factors in the development of psychopathology (pp. 181-214). New York, NY: Cambridge.

Santos, S. (2011). Versão Portuguesa do Parenting Stress Index (PSI) - Forma Reduzida: Estudo com uma amostra de mães de crianças com idade inferior a 5 anos. Poster apresentado no VIII Congresso Iberoamericano de Avaliação Psicológica/XV Conferência Internacional de Avaliação Psicológica: Formas e Contextos, Lisboa.

Saloviita, T., Itälinna, M., \& Leinonen, E. (2003). Explaining the parental stress of fathers and mothers caring for a child with intellectual disability: A double ABCX model. Journal of Intellectual Disability Research, 47(4/5), 300-312.

Seligman, M., \& Darling, R. B. (2007). Ordinary families, special children: A systems approach to childhood disability. New York, NY: The Guilford Press.

Seltzer, M. M., Greenberg, J. S., Floyd, F. J., Pettee, Y., \& Hong, J. (2001). Life course impacts of parenting a child with a disability. American Journal on Mental Retardation, 106(3), 282-303.

Shonkoff, J. P., Hauser-Cram, P., Krauss, M. W., \& Upshur, C. C. (1992). Development of infants with disabilities and their families: Implications for theory and service delivery. Monographs of the Society for Research in Child Development, 57(6), Serial No. 230, 1-153.

Stein, R. E., \& Jessop, D. J. (2003). The impact on family scale revisited: Further psychometric data. Developmental and Behavioral Pediatrics, 24(1), 9-16.

Svavarsdottir, E. K., \& Rayens, M. K. (2005). Hardiness in families of young children with asthma. Journal of Advanced Nursing 50(4), 381-390.

Taanila, A., Jarvelin, M. R., \& Kokkonen, J. (1999). Cohesion and parents' social relations in families with a child with disability or chronic illness. International Journal of Rehabilitation Research, 22(2), 101-109.

Tak, Y., \& McCubbin, M. (2002). Family stress, perceived social support and coping following the diagnosis of a child's congenital heart defect. Journal of Advanced Nursing, 39(2), 190-198.

Trute, B., \& Hauch, C. (1988). Building on family strength: A study of families with positive adjustment to the birth of a developmentally disabled child. Journal of Marital and Family Therapy, 14(2), 185-193.

Uzark, K., \& Jones, K. (2003). Parenting stress and children with heart disease. Journal of Pediatric Health Care, 17(4), 163-168.

Yeung, W. J., Sandberg, J. F., Davis-Kean, P. E., \& Hofferth, S. L. (2001). Children's time with fathers in intact families. Journal of Marriage and the Family, 63, 136-154. 